\title{
Accuracy and Multi Domain Piezoelectric Power Harvesting Model using VHDL-AMS and SPICE
}

\author{
Flavilene S.Souza, Nobuo Oki, Jozué V. Filho \\ Department of Electrical Engineering \\ UNESP/FEIS \\ Ilha Solteira/SP, Brasil \\ flavilene@hotmail.com
}

\author{
Richard Loendersloot, Arthur P. Berkhoff \\ Faculty of Engineering Technology \\ University of Twente \\ Enschede, Netherlands \\ r.loendersloot@utwente.nl
}

\begin{abstract}
This paper presents a piezoelectric power harvesting model including both the mechanical and electrical domain. It includes a mechanical system, electrical interface, storage capacitor and load. Bridge rectifier, Parallel Synchronized Switch Harvesting on Inductor (P-SSHI) and Synchronous Electric Charge Extraction (SECE) circuits are analyzed as electrical interface. A mechanical system and control signals are implemented in VHDL-AMS and electronics components in SPICE. Simulation results and experimental data are compared to show the accuracy of the proposed model.
\end{abstract}

Keywords - Power harvesting model; piezoelectric transducer; VHDL-AMS; SPICE; Electronic Circuit.

\section{INTRODUCTION}

Nowadays, there is a wide demand for autonomous systems. Generally, these systems use batteries as a power supply. In order to increase battery lifetime, self-powered sensors have been investigated. In this context, piezoelectric power harvesting (PPH) from mechanical vibration is an interesting topic [1]. It can be divided in 4 elements as shown in Fig. 1. The mechanical system is responsible for transforming a mechanical vibration into electrical energy (step 1). The output signal must be conditioned by an electrical interface (step 2) and stored (step 3). Then, the converted energy can be used at the load(step 4). It is usually a low power electronic device such as sensor and wireless transceiver [2].

Most PPH models are focused on the mechanical system or electrical interface separately. For the first, numerical analysis software is often utilized for modeling, while an Electronic Design Automation (EDA) is used for the electrical interface [3]. However, mechanical system responses are influenced by the electrical interface, and vice versa. This interaction affects the system performance, such as energy conversion. It means optimization should be based on a complete system model instead of separate components. In recent studies [3]-[6], analog and mixed-signal languages, like VHDL-AMS, are used to describe power harvesting system. However, they present a simple electrical interface. In this work, a whole PPH model using both VHDL-AMS and SPICE is proposed.

The analytical equation to describe the mechanical system is implemented in VHDL-AMS. For the electrical interface, SPICE and VHDL-AMS are used to model electronic components and control signals, respectively. Bridge rectifier,

CAPES

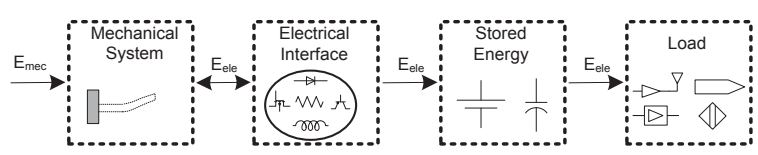

Fig. 2. Piezoelectric power havesting system from mechanical vibration.

P-SSHI and SECE circuits are used as electrical interface. Moreover, simulation results are shown and compared to experimental data.

\section{MECHANICAL SYSTEM}

A typical mechanical system of PPH from mechanical vibration consists of a cantilever beam and piezoelectric transducer (Fig. 2a). In this case, the maximum energy conversion is obtained by a maximum deformation of the piezoelectric. For this reason, $\mathrm{PPH}$ is often tuned to the resonance frequency. Around it, the mechanical system can be modeled as a single degree of freedom system (mass $(m)+$ spring $\left(k_{s}\right)+$ damper $(d)+$ transducer) [1], as shown in Fig. $2 \mathrm{~b}$. The function $z(t)$ refers to displacement of the mass, and $F_{P}(t), i_{P}(t)$ and $v_{P}(t)$ are the piezoelectric force, current and voltage, respectively. According to Newton's laws of motion, the system can be described as:

$$
F_{\text {in }}(t)=m \ddot{z}(t)+d \dot{z}(t)+k_{s} z(t)+F_{P}(t)
$$

Where $m=0.2235 m_{b}, \quad m_{b}=\rho_{b} w_{b} t_{b} l_{b}, \quad k_{s}=$ $E_{b} w_{b} t_{b}{ }^{3} / 4 l_{b}{ }^{3}$ and $d=\sqrt{k_{s} m} / Q . m_{b}$ is beam mass. Besides, $\rho_{b}, w_{b}, t_{b}, l_{b}$ and $E_{b}$ are density, width, thickness, length and Young's modulus of beam, respectively. In addition, $Q$ refers to the quality factor and $F_{i n}(t)$ is force applied to the system.

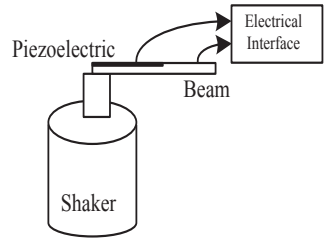

(a)

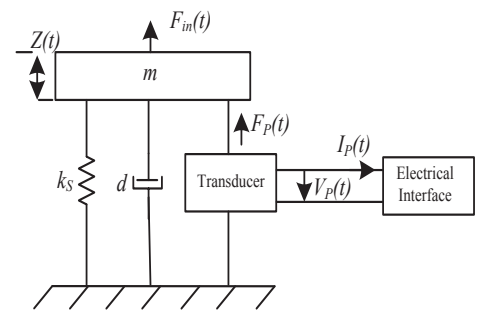

(b)
Fig. 1. A cantilever beam and piezoelectric transducer (a) and a single degree of freedom model (b) connected to electrical interface. 
Homogeneous stress and uniform electric field are assumed in the finite dimensional piezoelectric element. Under these conditions, the piezoelectric constitutive equations can be rewritten in terms of macroscopic variables as follows [2]:

$$
\left\{\begin{array}{l}
\mathrm{F}_{\mathrm{P}}(t)=k_{P} Z(\mathrm{t})+\alpha v_{P}(t) \\
i_{P}(t)=\alpha \dot{z}(t)-C_{P} \dot{v}_{P}(t)
\end{array}\right.
$$

For a piezoelectric excited in 31 mode, the variables in (2) are calculated similarly to [1]:

$$
\begin{aligned}
& k_{P}=\frac{A}{s_{11}^{E} l_{P}} \\
& \alpha=\frac{d_{33} A}{s_{11}^{E} l_{P}} \\
& C_{P}=\left(\varepsilon_{33}^{T}-\frac{d_{31}^{2}}{s_{11}^{E}}\right) \frac{A}{t_{P}}
\end{aligned}
$$

The variables $A, l_{P}$ and $t_{P}$ represent the cross-sectional area, length and thickness of the piezoelectric transducer, respectively. The piezoelectric properties are $s_{11}^{E}$ - elastic compliance under constant electric field, $d_{31}$ - piezoelectric constant and $\varepsilon_{33}^{T}$ - permittivity under constant stress.

Using (1) and (2), system behavior can be expressed as

$$
\left\{\begin{array}{c}
F_{\text {in }}(t)=m \ddot{z}(t)+d \dot{z}(t)+k z(t)+\alpha v_{P}(t) \\
i_{P}(t)=\alpha \dot{z}(t)-C_{P} \dot{v}_{P}(t)
\end{array}\right.
$$

where $k$ is a combination of the $k_{s}$ and $k_{P}$.

The differential equations in (6) are implemented in VHDL-AMS to describe the mechanical system. The model is shown in Fig. 3. The properties and dimension of the piezoelectric and the beam are input parameters of the model and $f_{\text {in }}(t)$ is a input force source. Furthermore, variables are set by two pairs of ports. $v_{P}(t)$ and $i_{P}(t)$ are defined as potential difference and flow through the electrical terminal (ET), respectively. Similarly, $z(t)$ and $f_{\text {in }}(t)$ are potential difference and flow across the translational terminal (TT), respectively. Thereby, the model obeys the energy conservation laws.

\section{ELECTRICAL INTERFACE}

The electrical interface is an electronic circuit responsible for signal conditioning. Considering a piezoelectric transducer excited by a sinusoidal vibration, $i_{P}$ is alternating current (AC). Usually, this current is not usable in electronic circuits. So $i_{P}$ must be converted to a direct current (DC).

A bridge rectifier (Fig. 3a) is the most common circuit. It is comprises of four diodes and a capacitor $C_{L}$ connected in parallel to the resistance $R_{L}$, which represents the load. In this analysis, ripple voltage is neglected. No control is required for this circuit. $C_{P}$ is charged in each semi-cycle. During this charging, all diodes are reverse biased and no current is delivered to the load. Once the $C_{P}$ has been charged, $i_{P}$ flows through the load. It limits the amount of load current $\left(i_{L O A D}\right)$ and, consequently, the average extracted power $\left(<\mathrm{P}_{L O A D}>\right)$. In order to improved it, some circuits have been proposed [7],[8], as P-SSHI and SECE.

P-SSHI (Fig. 3b), also known as bias-flip rectifier, is composed by a switched inductor ( $S$ and $L$ ) connected in parallel to piezoelectric and bridge rectifier. Switch $S$ is turned

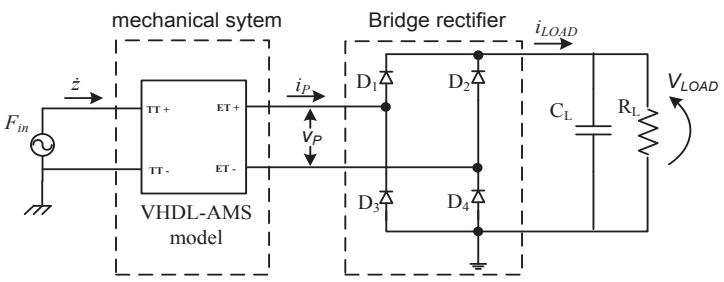

(a)

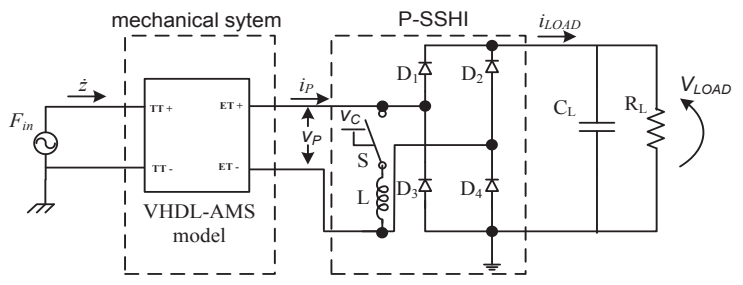

(b)

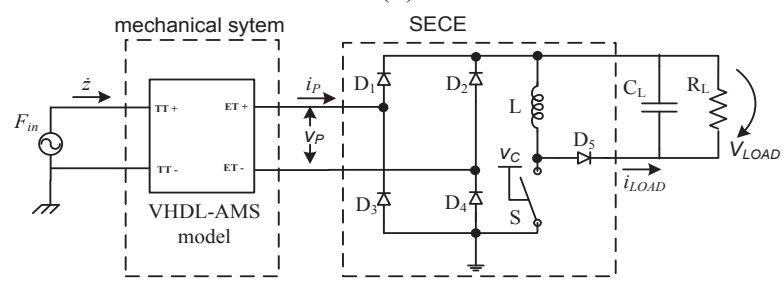

(c)

Fig. 3. Electrical interface examples: (a) Bridge rectifier; (b) Parallel Synchronized Switch Harvesting on Inductor (P-SSHI); (c) Synchronous Electric Charge Extraction (SECE).

on when the polarity of $i_{p}$ is reversed (similarly $v_{P}$ starts to decrease or increase). An $L-C_{P}$ electronic oscillator circuit is established. Ideally, it leads to a quasi-instantaneous $v_{P}$ inversion. In other words, it will charge the $C_{P}$. Then $S$ is turned off and $i_{P}$ flows through the load. Therefore, $i_{L O A D}$ in P$\mathrm{SSHI}$ is greater than in bridge rectifier. This increases $\left\langle\mathrm{P}_{L O A D}>\right.$.

SECE (Fig. 3c) is composed by the DC-DC converter connected between bridge rectifier and load. Switch $S$ is closed at the moment $v_{P}$ achieves a maximum or minimum value. Thus, the energy stored on $C_{P}$ is transferred to $L$ until $v_{P}$ go to zero. Then $\mathrm{S}$ is turned off and the inductor current flows through the load. Besides, $C_{P}$ is being charged. Consequently, load is not connected directly to piezoelectric transducer.

Control signals $v_{c}$ are required for the P-SSHI and SECE simulation. In this work, a behavioral model is used to obtain the control signals. They are determined from observation of $v_{P}$. SPICE is an industry standard used to analyze. However, it does not allow the use of behavioral models. Therefore, in this work, the SPICE is used for electronics components and VHDL-AMS for control.

\section{RESULTS}

A piezoelectric P-876 A11 DuraAct Patch Transducer excited in 31 mode was used, bonded on an aluminum cantilever beam with dimension of $35.0 \times 200.5 \times 2.0 \mathrm{~mm}^{3}$. Simulations were carried out in SystemVision Software of Mentor Graphics because it allows simultaneous use of VHDLAMS and SPICE. The applied force was a sine wave with amplitude of $100 \mathrm{mg}$. In experimental setup, the beam was 


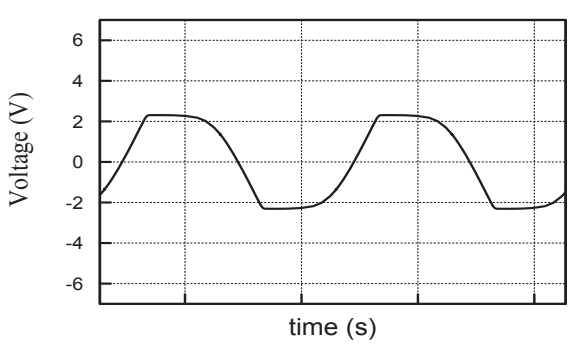

(a)

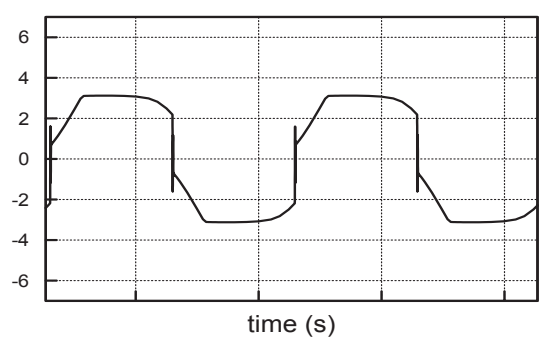

(b)

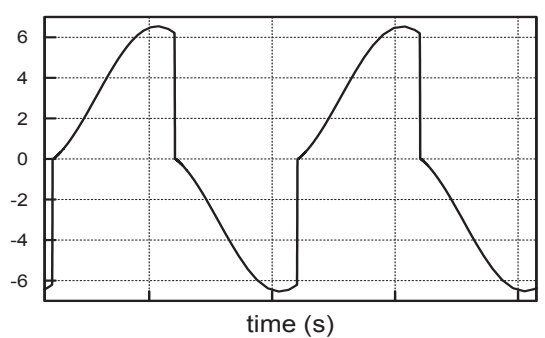

(c)

Fig. 4. Piezoelectric voltage waveforms at resonance frequency and load of $68 \mathrm{~K} \Omega$ for: (a) Bridge rectifier; (b) P-SSHI; (c) SECE.

excited by a shaker. Frequency and amplitude were controlled by an acquisition system (NI 4431 from National Instruments) and a power amplifier (type 2706 from Bruel \& Kjaer). Also, the schottky diodes and the inductor Bourns SDR1005-102KL were used. And the control signals were generated by arduino microcontroller.

In this work by default, the excitation frequency and load were $30.0 \mathrm{~Hz}$ (resonance frequency) and $68 \mathrm{~K} \Omega$, respectively. Fig. 4 shows piezoelectric voltage waveforms for the bridge rectifier, P-SSHI and SECE circuits. In the bridge rectifier, $v_{P}$ was equal to the charge of $C_{P}$. At the moment the control signal was high, in P-SSHI the polarity of $v_{P}$ was inverted, but the flip was not complete due the equivalent series resistance (ESR) of the inductor. Meanwhile, in SECE it went to ground when the control signal was high. It is consistent as showed in [7] and described.

The average extracted power as a function of the excitation frequency and load resistance are shown in Fig. 5. Lines and

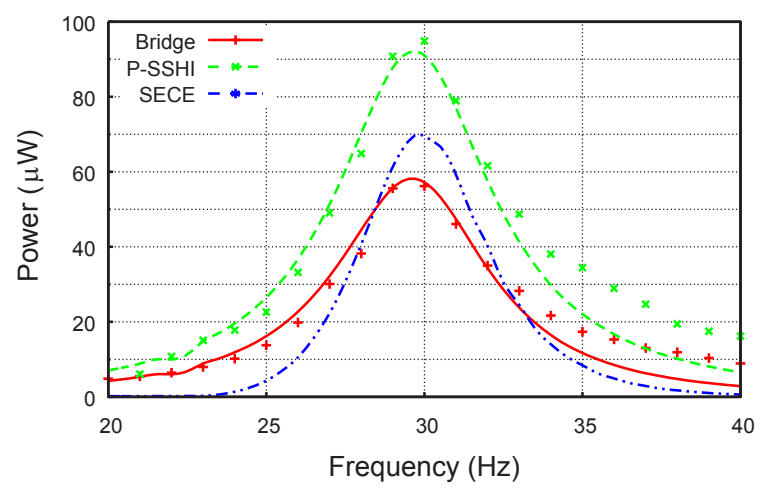

(a)

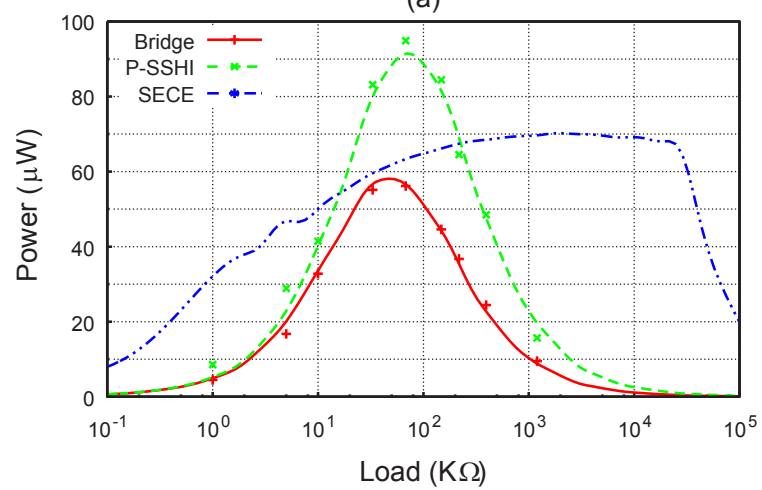

(b)

Fig. 5. Average extracted power for bridge rectifier, P-SSHI and SECE as a fuction of: (a) excitation frequency; (b) load resistance. points represent to simulation results and experimental data, respectively. Comparison between both shows the simulation prediction is quite close to the experimental results. It shows the model is accuracy. In addition, results showed the average extracted power was dependent of frequency and load. Considerable power was obtained around the resonance frequency $(30.0 \mathrm{~Hz})$. Moreover, P-SSHI and SECE were able to provide $160 \%$ and $123 \%$ more power than the bridge rectifier, respectively. More important, bandwidth was improved by $110 \%$ in P-SSHI compared to the bridge rectifier. Significant amount of power was obtained in a resistance range. The SECE has better load range than the other circuits.

\section{CONCLUSION}

A piezoelectric power harvesting model using VHDLAMS and SPICE was proposed. Results show the model is working properly and accurately. In other words, it is adequate to predict the whole system behavior. The model advantage is the ability to execute simulations for various excitation frequency in SPICE using only the properties and dimension of beam and piezoelectric as input. Thus, the model is an important tool to design and optimize piezoelectric power harvesting system in both mechanical and electrical domain.

\section{REFERENCES}

[1] P. Spies, L. Mateu, and M. Pollak, Eds., Handbook of Energy Harvesting Power Supplies and Applications. Taylor \& Francis Group, 2013.

[2] T. Hehn and Y. Manoli, CMOS Circuits for Piezoelectric Energy Harvesters: Efficient Power Extraction, Interface Modeling and Loss Analysis, 1st ed., vol. 38. Heidelberg: Springer Netherlands, 2015.

[3] T. J. Kaźmierski and S. Beeby, Eds., Energy Harvesting Systems, 1st ed. New York: Springer-Verlag New York, 2011.

[4] H. Boussetta, M. Marzencki, Y. Ammar, and S. Basrour, "Multilevel modeling of integrated power harvesting system using VHDL-AMS and SPICE," in IEEE Int. Behavioral Modeling Simulations Workshop, San Jose, CA, USA, 2007, pp. 126-131.

[5] H. Durou, et al., "Power harvesting and management from vibrations: a multi-source strategy simulation for aircraft structure health monitoring," in Proc. SPIE 7268, Smart Structures, Devices, and Systems IV, Melbourne, Australia, 2008, pp. 726810.

[6] L. Mateu and F. Moll, "System-level simulation of a self-powered sensor with piezoelectric energy harvesting," in Int. Conf. Sensor Technologies Applications,. SENSORCOMM 2007,Valencia, Spain, 2007, pp. 399-404.

[7] E. Lefeuvre, A. Badel, C. Richard, L. Petit, and D. Guyomar, "A comparison between several vibration-powered piezoelectric generators for standalone systems," Sensors and Actuators, A: Physical, vol. 126, no. 2, [s.i], pp. 405-416, 2006.

[8] S. Pang, W. Li, and J. Kan, "Optimization Analysis of Interface Circuits in Piezoelectric Energy Harvesting Systems," J. Power Technol., vol. 96, no. 1, pp. 1-7, 2016. 